\title{
Consumo de suplementos alimentares por praticantes de exercícios físicos
}

\author{
Consumption of dietary supplements by exercise practitioners \\ Consumo de suplementos dietéticos por parte de los practicantes de ejercicio
}

Recebido: 14/07/2021 | Revisado: 21/07/2021 | Aceito: 22/07/2021 | Publicado: 31/07/2021

\author{
Givanildo de Oliveira Santos \\ ORCID: https://orcid.org/0000-0001-5279-1535 \\ Faculdade de Mauá Brasília, Brasil \\ E-mail: givanildo-o@hotmail.com \\ Lairon Freitas de Paula \\ ORCID: https://orcid.org/0000-0003-1340-8518 \\ Faculdade de Piracanjuba, Brasil \\ E-mail: laironfreitas@hotmail.com \\ Anarelle Freitas de Paula \\ ORCID: https://orcid.org/0000-0001-8767-9121 \\ Faculdade de Piracanjuba, Brasil \\ E-mail: sharmemodas@ hotmail.com \\ Sebastião Lobo da Silva \\ ORCID: https://orcid.org/0000-0003-2639-0898 \\ Universidade Católica de Brasília, Brasil \\ E-mail: slobo2011@gmail.com
}

\begin{abstract}
Resumo
Objetivou-se verificar o perfil dos principais consumidores de suplementos alimentares, os principais suplementos consumidos e a prática de exercícios físicos associada ao consumo, bem como os principais profissionais que indicam a suplementação. Para a realização deste estudo, pesquisou-se artigos científicos em revistas e periódicos nacionais e internacionais que tratavam do tema proposto. Após a realização da revisão sobre o assunto, pode-se concluir que a maioria das pessoas consomem suplementos alimentares sem a indicação de um profissional capacitado, e concomitante a este fato, poderá desencadear sérios problemas a saúde. Pode-se verificar que ambos os gêneros consumem suplementos alimentares com objetivos afins.
\end{abstract}

Palavras-chave: Musculação; Treinamento resistido; Nutrição; Nutracêuticos; Academias.

\begin{abstract}
The objective was to verify the profile of the main consumers of dietary supplements, the main supplements consumed and the practice of physical exercises associated with consumption, as well as the main professionals who indicate supplementation. In order to carry out this study, scientific articles were searched in national and international journals and journals that dealt with the proposed theme. After conducting the review on the subject, it can be concluded that most people consume food supplements without the indication of a trained professional, and concomitant to this fact, it can trigger serious health problems. You can see that both sexes consume food supplements for similar purposes.
\end{abstract}

Keywords: Bodybuilding; Resistance training; Nutrition; Nutraceuticals; Gyms.

\section{Resumen}

El objetivo fue verificar el perfil de los principales consumidores de complementos alimenticios, los principales complementos consumidos y la práctica de ejercicio físico asociado al consumo, así como los principales profesionales que indican la suplementación. Para la realización de este estudio se investigaron artículos científicos en revistas y revistas nacionales e internacionales que abordaron el tema propuesto. Tras realizar una revisión sobre el tema, se puede concluir que la mayoría de personas consumen complementos alimenticios sin la indicación de un profesional capacitado, y concomitante con este hecho, puede desencadenar graves problemas de salud. Puedes ver que ambos sexos consumen complementos alimenticios con objetivos similares.

Palabras clave: Culturismo; Entrenamiento de resistencia; Nutrición; Nutracéuticos; Gimnasios.

\section{Introdução}

Suplementos nutricionais são componentes alimentares, por exemplo, proteína, e não alimentos ou preparações farmacêuticas, por exemplo vitaminas e minerais em cápsulas ou comprimidos, fornecendo um ou mais nutrientes em forma 
concentrada, incluindo proteínas, minerais, vitaminas e outros componentes que estão teoricamente presentes em dieta normal e balanceada (De Oliveira, et al., 2019).

Os suplementos nutricionais são geralmente oferecidos em forma atípica de alimentos, incluindo comprimidos, cápsulas, pós ou comprimidos. Embora muitos indivíduos usem suplementos, descobriu-se que aqueles que praticam esportes e atividades físicas representam a maior parte dos compradores suplementos (Maughan, et al., 2004).

Há evidências suficientes de que pessoas fisicamente ativas não requerem nutrientes adicionais, além daqueles obtidos por meio de uma dieta balanceada (Smith-rockwell, et al., 2001). A American Dietetic Association, os Dietitians of Canada e o American College of Sports Medicine declararam que apenas aquelas pessoas que restringem sua ingestão de energia, usam práticas severas de perda de peso, eliminam um ou mais grupos de alimentos de suas dietas ou consomem dietas ricas em carboidratos com baixa densidade de micronutrientes pode exigir suplementação alimentar (Huang, et al., 2005).

$\mathrm{Na}$ verdade, a indústria de suplementos nutricionais é atualmente um mercado internacional que vale bilhões de dólares (Van thuyne, et al., 2006). Nos Estados Unidos, relatou-se que mais de 3 milhões de pessoas usavam ou usaram suplementos ergogênicos (Palmer, et al., 2003).

Há um uso crescente de suplementos nutricionais por pessoas envolvidas em atividades físicas ou atléticas (Calfee \& Fadale, 2006). O uso de suplementos entre atletas é bem documentado. Estima-se que varia de 40 a $88 \%$ (Sobal \& Marquart, 1994), e varia de acordo com os tipos de esportes, aspectos culturais, faixas etárias e gênero (Maughan, et al., 2004).

O aumento na demanda por suplementos nutricionais foi atribuído a várias razões, incluindo melhorar o desempenho, melhorar a saúde, prevenir deficiências nutricionais e doenças, aumentar a massa muscular, diminuir a gordura corporal, aumentar a imunidade, aumentar o estado de alerta e a atividade mental, melhorar a recuperação e reduzir o estresse (Erdman, et al., 2006).

No entanto, os benefícios exatos dos suplementos nutricionais ainda não estão bem estabelecidos (Volpe, 2007). Além disso, vários riscos potenciais foram descritos ao usar suplementos nutricionais em altas doses ou sem supervisão médica (Schwenk \& Costley, 2002).

Objetivou-se verificar o perfil dos principais consumidores de suplementos alimentares, os principais suplementos consumidos e a prática de exercícios físicos associada ao consumo, bem como os principais profissionais que indicam a suplementação.

\section{Metodologia}

De acordo com Estrela (2018), a presente pesquisa trata-se de uma revisão bibliográfica de natureza qualitativa, integrando estudos já concluídos e realizando uma discussão a partir dos resultados encontrados, tendo critérios de inclusão e exclusão. Entretanto, para o refinamento adequado de pesquisa, foi definida uma amostra, obedecendo aos seguintes critérios de inclusão: publicações em português e em inglês que abordaram a temática, atendendo as palavras-chave: "creatina", "suplementos alimentares", "treinamento resistido e suplementação", que utilizaram algum percurso metodológico de pesquisa experimental em protocolos avaliados com a suplementação e a prática de treinamento resistido e outras modalidades esportivas. As pesquisas por artigos foram realizadas nas plataformas de pesquisas: PubMed, Lilacs, Google acadêmico, MedLine, ScienceDirect, SciELO, ScienceDirect e Elsevier. 


\section{Revisão}

\subsection{Consumidores de suplementos alimentares}

Em meio as possíveis relações de aumento do consumo de suplementos alimentares, podem ocorrer através das falsas promessas apresentadas em sua rotulagem como: aumentar a energia, aliviar o estresse, aumentar o desempenho e prevenir ou tratar doenças. Mesmo não havendo comprovação cientifica por muitos produtos vendidos que podem gerar danos à saúde dos consumidores, quando consumidos de forma inadequada e sem orientação de um profissional capacitado (De Oliveira, 2011).

Em Campo Grande atingiu 56\%, o consumo de suplementos alimentares entre os profissionais de Educação Física Florianópolis 44\% (Coelho, et al., 2007). Em que a prevalência de prescrição dos suplementos não foi realizada por um profissional capacitado.

De acordo com Menon, et al (2012), descreve que para o ganho de massa muscular, a alimentação é ferramenta fundamental, obtendo a relevância de $60 \%$ para este objetivo. Porém, a falta de informação para os consumidores, que a alimentação saudável fornece os macros e micronutrientes que atendem as necessidades dos atletas, no entanto, em alguns casos, os atletas de alto rendimento precisam de uma suplementação à base de proteínas (Erdman, et al., 2006).

Conforme relata estudo de Goston \& Correia (2010), 36,8\% dos praticantes de exercícios físicos em academias na cidade de Belo Horizonte consomem suplementos alimentares. Portanto, as taxas observadas na cidade de Nova York (84,7\%) e na Espanha $(56,1 \%)$ entre os praticantes de exercícios físicos são maiores que em Belo Horizonte (Oliver, et al., 2008).

O principal alvo do mercado de suplementos alimentares são os praticantes de exercícios físicos em academias, porém, em vários estudos relatam que a maior parte dos consumidores de suplementos alimentares são atletas (Erdman, et al., 2006).

Portanto, existe discrepância nas taxas relatadas, e, podendo explicar estas diferenças as academias incluídas nos estudos, os participantes, a forma de coletas de dados, e a falta de conhecimento em definir o que realmente são suplementos alimentares (Froiland, et al., 2004).

Os perfis dos consumidores podem ser diferentes por gênero sexual e tipo de exercício físico praticado. Em estudo de Goston \& Correia (2010), o consumo de suplementos alimentares se apresentou com maior frequência entre praticantes do gênero masculinos e atletas adolescentes. Por outro lado, Sobal \& Marquart (1994), relataram a prevalência maior de consumidores de suplementos entre o sexo feminino. No entanto, Sundgot-Borgen et al (2003), não reportaram diferença significativa entre os gêneros sexuais. No quadro 1. Atividades esportiva e consumo de suplementos alimentares, podendo verificar algumas das atividades esportivas praticadas e principais suplementos ingeridos para a prática dos mesmos. 
Quadro 1. Atividades esportiva e consumo de suplementos alimentares.

\begin{tabular}{|c|c|c|c|}
\hline 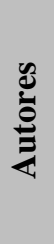 & 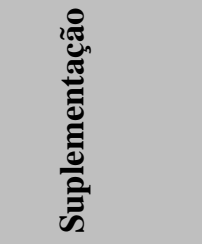 & Consumo & $\begin{array}{l}\text { Tipo } \\
\text { Exercício Físico }\end{array}$ \\
\hline 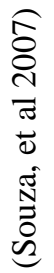 & Creatina & $\begin{array}{l}\text { Fase } 1=30 \mathrm{~g} \text { de creatina monohidratada, dividida em } 5 \text { vezes ao dia. } \\
\text { Fase } 2 \text { ou manutenção }=5 \mathrm{~g} \text { de creatina monohidratada por dia. }\end{array}$ & Treino de força \\
\hline 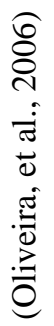 & $\begin{array}{l}\text { Whey } \\
\text { Protein }\end{array}$ & $\begin{array}{l}\text { Grupo } \mathrm{HP}=4 \mathrm{~g} \text { de proteína do soro do leite por quilo de peso } \\
\text { corporal; Grupo NP }=1,6 \mathrm{~g} \text { de PTN e maltodextrina por quilo de peso } \\
\text { de corpo corporal. }\end{array}$ & $\begin{array}{l}\text { Treino de força } \\
\text { (hipertrofia) }\end{array}$ \\
\hline 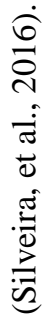 & Glutamina & $\begin{array}{l}\text { Fase de adaptação: } 3 \mathrm{~g} . \mathrm{kg} / \text { dia de peso corporal, dividido em } 3 \text { doses } \\
\text { diárias. Fase de Manutenção: } 3 \mathrm{~g} . \mathrm{kg} / \text { dia de peso corporal, em dose } \\
\text { única, } 30 \text { min após a sessão. }\end{array}$ & Treino de força \\
\hline 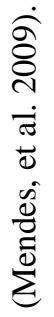 & Carboidratos & $\begin{array}{l}3 \text { ml.kg de peso corporal da bebida carboidratada a cada } 15 \text { minutos } \\
\text { tanto durante a sessão de treino, quanto na sessão de descanso. }\end{array}$ & Judô \\
\hline 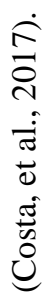 & Carboidratos & $\begin{array}{l}\text { Foram ofertadas de } 5 \text { a } 8 \mathrm{~g} \text { de carboidrato por quilo de massa corporal } \\
\text { para as atletas durante cada dia da semana, além de suplementação de } \\
\text { bebida carboidratada, com concentração de } 30 \mathrm{~g} \text { de carboidratos } \\
\text { durante as sessões de treinamento. }\end{array}$ & $\begin{array}{l}\text { Ginástica } \\
\text { Rítmica }\end{array}$ \\
\hline
\end{tabular}

Fonte: Autores.

\subsection{Suplementos alimentares mais consumidos}

Em estudo de Goston e Correia (2010), relataram que homens e jovens praticantes de exercícios físicos continham a maior probabilidade de consumir suplementos alimentares proteicos e aminoácidos de cadeia ramificada, e as mulheres e 
praticantes com idade acima de 40 anos consumiam suplementos alimentares ricos em vitaminas, minerais e fitoterápicos. Em estudo de Froiland, et al (2004), relataram que as praticantes de exercícios físicos universitárias femininas consumiam com maior frequência cálcio e multivitaminas, por outro lado, os homens consumiam os aminoácidos, glutamina e proteína de soro de leite.

De acordo com Goston e Correia (2010), praticantes de exercícios físicos com menos de 30 anos consumiram os suplementos alimentares objetivando o aumento da massa muscular, já os praticantes com idade acima de 45 anos consumiam suplementos objetivando a prevenção de patologias. Isso foi relatado por Tian, et al., (2009), que relataram o uso de multivitaminas, vitamina $\mathrm{C}$ e produtos fitoterápicos foram maiores entre as atletas universitárias em Cingapura objetivando benefícios à saúde, por outro lado, atletas consumiram com o objetivo de ganhar massa muscular, consumindo proteínas e aminoácidos.

A grande maioria dos consumidores de suplementos alimentares não obtém o conhecimento dos impactos negativos que os suplementos alimentares podem ocasionar na saúde, quando consumido de maneira inadequada. Em estudo de Tian, et al., (2009), em praticantes de exercícios físicos universitários em Cingapura, 86,4\% não sabiam que o consumo exagerado e de uso inadequado poderão influenciar na saúde, causando alguma patologia ou distúrbios metabólicos.

Normalmente, estas informações são atribuídas as fontes indevidas de informações que são repassadas aos praticantes de exercícios físicos e consumidores de suplementos alimentares, e $80 \%$ dos consumidores e praticantes de exercícios físicos obtém informações da mídia, internet, e instrutores de exercícios físicos (Slater, et al., 2003).

Conforme Brasil, et al., (2009), o suplemento alimentar comumente de maior consumo pelos entrevistados em pesquisa foram os suplementos proteicos com 65,9\%. Portanto, Ferreira, et al., (2003), relataram que os suplementos proteicos com $30,77 \%$, e o carboidrato sendo o suplemento alimentar com maior consumo. Portanto, em estudo de Petroczi \& Naughton (2008), descreveram que os suplementos mais consumidos foram os multivitamínicos com o percentual de 72,6\%, seguido por vitamina C com 70,7\%, a creatina com $36,1 \%$ e a por último a Whey Protein reportada com $31,7 \%$.

Ferreira, et al., (2003), relataram que 17,18\% da sua população estudada consumiam algum suplemento alimentar. Diferente de Domingues e Marins (2007), que estudaram 200 praticantes de musculação, e 48\% reportaram consumir suplemento de maneira contínua e $30 \%$ sem continuidade.

No entanto, Milani, et al., (2014), relataram que os suplementos mais consumidos por praticantes de musculação foram os ricos em proteínas com 33,0\%, os aminoácidos $18,0 \%$, e creatina $14,0 \%$, ricos em carboidratos $8,0 \%$ e os queimadores de gordura 7,0\%. Porém, em trabalho realizado por Costa (2013), relataram que o suplemento mais consumido com $72,0 \%$ os aminoácidos e concentrados proteicos, seguido de 4,0\% creatina, 4,0\% termogênico, 4,0\% hipercalórico e $12,0 \%$ carboidratos. Em pesquisa realizada em Porto Alegre - RS, os avaliados reportaram consumir suplementos proteicos $38,9 \%$, suplementos calóricos 21,3\%) e de vitaminas ou minerais (12,3\%) (Fayh, et al., 2013).

\subsection{Prescrição dos suplementos alimentares}

Devido a oferta de suplementos alimentares, os praticantes de exercícios resistidos utilizam os mesmos sem as devidas orientações profissionais, e sem saber que este fato poderá acarretar complicações e consequências à saúde (Moya, et al., 2009).

Os entrevistados quando questionados quem os orientavam para a compra dos suplementos alimentares, foi relatado que $33,33 \%$ se auto prescreviam; $29,17 \%$ faziam acompanhamento com nutricionista; $25 \%$ eram indicados por profissionais de Educação Física; e 12,5\%, por amigos (Moya, et al., 2009).

No entanto, Pereira, et., al (2003), relataram que os profissionais que mais faziam as recomendações de suplementos alimentares, eram os professores de Educação Física 31,1\%, amigos 15,6\%, auto indicação 15,6\%, nutricionista 11,1\% e 
médico 10,0\%. Estes autores acrescentaram e alertaram que os próprios professores vendem esses suplementos e mesmo sem ter a formação científica.

Santos, et al., (2013), relataram que 37,0\% eram orientados por amigos e $27,0 \%$ por instrutor ou professor de Educação Física. Já em estudo realizador por Frade, et al., (2016), a autoprescrição e por nutricionista se mantiveram em 28,9\%, por amigos 18,4\%, professional de Educação Física 7,9\%, vendedor loja 2,6\% e fisioterapeuta 0,9\%. Por outro lado, em pesquisa realizada 54,0\% consumiam suplementos alimentares sem indicação de um profissional habilitado e 46,0\% por professores de Educação Física (Araujo \& Soares, 1999).

De Oliveira, et al., (2019), descreve que o consumo de suplementos alimentares deve ser associado à dieta e exercícios físicos, visto que os profissionais de nutrição relatam que o treino associado a uma dieta equilibrada, melhora o desempenho físico e esportivo; sugerindo o consumo da suplementação alimentar por apenas atletas e em casos específicos de deficiência nutricional, sempre acompanhados e prescritos por profissional capacitados.

\subsection{Objetivo do consumo de suplementos}

Devido os praticantes de exercícios físicos objetivar corpo perfeito e a melhora do desempenho físico, muita das vezes têm influenciado as pessoas a consumirem recursos ergogênicos que potencializam os objetivos em menor período. Entre estes recursos podem se destacar os suplementos alimentares que, podendo ser comprados sem receita médica e sem quaisquer prescrições (Zeiser \& Silva, 2007).

Mesmo com os suplementos alimentares sendo utilizados cada vez mais, pode-se notar que há grande falta de informações por pessoas que compram para consumo, demonstrando a facilidade de compreender diversas matérias em veículos de comunicação reportando várias dúvidas com relação ao consumo de suplementos alimentares, desse modo, fazendo o consumo dos suplementos alimentares seja feita de modo pouco consciente (Bacurau, 2005).

Conforme pesquisa realizada por Pereira, et al., (2003), os praticantes de exercícios físicos consumiam suplementos alimentares objetivando aumentar o desempenho físico, aumento de massa muscular. Portanto, o consumo de suplementos alimentares está relacionado a melhoria no desempenho físico (Santos \& Santos, 2002).

Pedrosa, et al., (2011) relataram que a maioria dos consumidores de suplementos alimentares obtiveram mudanças, como hipertrofia $42,0 \%$, ganho de força $28,0 \%$, e maior disposição ao trabalho $23,0 \%$.

\section{Considerações Finais}

Como os suplementos nutricionais não justifica as más escolhas nos hábitos alimentares inadequados, desse modo não isentando os riscos à saúde, tornando-se extremamente essencial a divulgação de informações cientificamente sobre o consumo adequado dos suplementos alimentares, potencializando os benefícios e minimizando os possíveis efeitos colaterais a saúde.

Os suplementos mais consumidos por participantes de exercícios físico são à base de proteína e carboidratos, porém, a maioria destes consumidores compra os suplementos alimentares por iniciativa própria, sem nenhuma orientação de um profissional qualificado. Entretanto, na maioria dos casos, os maiores influenciadores pela compra e ingestão são os profissionais de Educação Física, amigos e vendedores de lojas de suplementos alimentares.

Pode ser observado também que os consumidores de suplementos alimentares eram de ambos os sexos, tendo como atividade física mais praticada o treinamento resistido. Quando verificado quanto ao objetivo no consumo destes suplementos, a maioria das pesquisas relataram que os consumidores objetivam o desempenho físicos e ganho de massa muscular.

Após o levantamento bibliográficos realizados de natureza qualitativa, sugerimos para futuras pesquisas uma revisão sistematizada de natureza quantitativa, para que possa mensurar estatisticamente os resultados de estudos aplicados a 
Research, Society and Development, v. 10, n. 9, e46310918261, 2021

(CC BY 4.0) | ISSN 2525-3409 | DOI: http://dx.doi.org/10.33448/rsd-v10i9.18261

voluntários em pesquisas com intervenção.

\section{Referências}

Araújo, A. C. M. D., \& Soares, Y. D. N. G. (1999). Perfil de utilização de repositores proteicos nas academias de Belém, Pará. Revista de Nutrição, 12, 81-89.

Brasil, T. A., Pinto, J. A., Cocate, P. G., Chácara, R. P., \& Marins, J. C. B. (2009). Avaliação do hábito alimentar de praticantes de atividade física matinal. Fitness \& Performance Journal, (3), 153-163

Calfee, R., \& Fadale, P. (2006). Popular ergogenic drugs and supplements in young athletes. Pediatrics, 117(3), e577-e589.

Coelho, C. D. F., Camargo, V. R., \& Ravagnani, F. C. (2007). Consumo de suplementos nutricionais por praticantes de musculação em academia de Campo Grande, MS. Rev Nut Pauta, 15(87), 41-6.

Costa, D. C., Rocha, N. C. A., \& Quintão, D. F. (2013). Prevalência do uso de suplementos alimentares entre praticantes de atividade física em academias de duas cidades do Vale do Aço/MG: fatores associados. RBNE-Revista Brasileira de Nutrição Esportiva, 7(41).

Costa, T. A., Gonçalves, H. R., Anschau, F. R., Viaro, L. F., Borgheti, R., Santos, F. B. D., \& Borges, J. H. (2017). Suplementação com bebida artesanal que contém carboidrato em atletas da ginástica rítmica. Revista Brasileira de Ciências do Esporte, 39, 115-122.

De Oliveira Phillipps, C. (2011). Prevalência do uso de suplementos nutricionais pelos praticantes de atividade física, clientes de uma loja de suplementos. Revista Brasileira de Nutriçao Esportiva, 5(26), 3.

De Oliveira Santos, G., Daciuk, L. M. O. M., de Souza, R. F., \& de Oliveira Bozelli, R. Uso consciente de suplementos alimentares. Uso consciente de suplementos alimentares, 1-388.

Estrela, C. (2018). Metodologia Científica: Ciência, Ensino, Pesquisa. Editora Artes Médicas.

Domingues, S. F., \& Marins, J. C. B. (2007). Utilização de recursos ergogênicos e suplementos alimentares por praticantes de musculação em Belo HorizonteMG. Fitness \& performance journal, 6(4), 218-226.

Erdman, K. A., Fung, T. S., \& Reimer, R. A. (2006). Influence of performance level on dietary supplementation in elite Canadian athletes. Medicine and science in sports and exercise, 38(2), 349-356.

Fayh, A. P. T., Silva, C. V. D., Jesus, F. R. D. D., \& Costa, G. K. (2013). Consumo de suplementos nutricionais por frequentadores de academias da cidade de Porto Alegre. Revista Brasileira de Ciências do Esporte, 35, 27-37.

Ferreira, S., Lunz, W., Viana, E. C., Da Silva, L. C., \& Silva, A. G. (2003). Perfil e nível de conhecimento sobre nutrição em usuários de academia. In Anais do $7^{\circ}$ Congresso da Sociedade Brasileira de Alimentação e Nutrição-SBAN. Belo Horizonte.

Frade, R. E., Viebig, R. F., \& Fonseca, D. C. (2016). Avaliação do consumo de suplementos nutricionais por frequentadores de uma academia da cidade de São Paulo-SP. RBNE-Revista Brasileira de Nutrição Esportiva, 10(55), 50-58.

Froiland, K., Koszewski, W., Hingst, J., \& Kopecky, L. (2004). Nutritional supplement use among college athletes and their sources of information. International journal of sport nutrition and exercise metabolism, 14(1), 104-120.

Goston, J. L., \& Correia, M. I. T. D. (2010). Intake of nutritional supplements among people exercising in gyms and influencing factors. Nutrition, 26(6), 604611.

Hallak, A., Fabrini, S. P., \& Peluzio, M. D. C. G. (2007). Avaliação do consumo de suplementos nutricionais em academias da zona sul de Belo Horizonte, MG, Brasil. Revista Brasileira de Nutrição Esportiva, 1(2), 7.

Harrison, R. A., Holt, D., Pattison, D. J., \& Elton, P. J. (2004). Are those in need taking dietary supplements? A survey of 21923 adults. British Journal of Nutrition, 91(4), 617-623.

Huang, S. H. S., Johnson, K., \& Pipe, A. L. (2006). The use of dietary supplements and medications by Canadian athletes at the Atlanta and Sydney Olympic Games. Clinical Journal of Sport Medicine, 16(1), 27-33.

Maughan, R. J., King, D. S., \& Lea, T. (2004). Dietary supplements. Journal of sports sciences, 22(1), 95-113.

Mendes, E. L., Brito, C. J., Batista, E. S., Silva, C. H. O., Paula, S. O. D., \& Natali, A. J. (2009). Influência da suplementação de carboidrato na função imune de judocas durante o treinamento. Revista Brasileira de Medicina do Esporte, 15, 58-61.

Milani, V. C., Teixeira, E. L., \& Marquez, T. B. (2014). Fatores associados ao consumo de suplementos nutricionais em frequentadores de academias de ginástica da cidade de Nova Odessa. Revista Brasileira de Nutrição Esportiva, 8(46), 9.

Moya, R. N., Seraphim, R. V., Calvano, J. C., \& de Oliveira Alonso, D. (2009). Utilização de suplementos alimentares por adultos jovens, praticantes de musculação use of nutritional supplements by young adults, bodybuilders. Revista de Atenção à Saúde, 7(19).

Oliveira, P. V. D., Baptista, L., Moreira, F., \& Lancha Junior, A. H. (2006). Correlação entre a suplementação de proteína e carboidrato e variáveis antropométricas e de força em indivíduos submetidos a um programa de treinamento com pesos. Revista Brasileira de Medicina do Esporte, $12,51-55$.

Oliver, A. J., Leon, M. T., \& Hernández, E. G. (2008). Statistical analysis of the consumption of nutritional and dietary supplements in gyms. Archivos latinoamericanos de nutricion, 58(3), 221-227. 
Research, Society and Development, v. 10, n. 9, e46310918261, 2021

(CC BY 4.0) | ISSN 2525-3409 | DOI: http://dx.doi.org/10.33448/rsd-v10i9.18261

Palmer, M. E., Haller, C., McKinney, P. E., Klein-Schwartz, W., Tschirgi, A., Smolinske, S. C., \& Landzberg, B. R. (2003). Adverse events associated with dietary supplements: an observational study. The Lancet, 361(9352), 101-106.

Pedrosa, O. P., Qasen, F. B., da Silva, A. C., \& de Pinho, S. T. (2011). Utilização de suplementos nutricionais por praticantes de musculação em academias da cidade de Porto Velho Rondônia. Anais seminário nacional educa, 1(1).

Pereira, R. F., Lajolo, F. M., \& Hirschbruch, M. D. (2003). Consumo de suplementos por alunos de academias de ginástica em São Paulo. Revista de Nutrição, 16, 265-272.

Petroczi, A., \& Naughton, D. P. (2008). The age-gender-status profile of high performing athletes in the UK taking nutritional supplements: lessons for the future. Journal of the International Society of Sports Nutrition, 5(1), 1-8.

Santos, H. D., de Oliveira, C. C. P., de Freitas, A. K. C., \& Navarro, A. C. (2013). Consumo de suplementos alimentares por praticantes de exercício físico em academias de bairros nobres da cidade do Recife. RBNE-Revista Brasileira De Nutrição Esportiva, 7(40).

Dos Santos, M. Â. A., \& dos Santos D, R. P. (2002). Uso de suplementos alimentares como forma de melhorar a performance nos programas de atividade física em academias de ginástica. Rev. paul. Educ. Fís., São Paulo, 16(2), 174-85.

Schwenk, T. L., \& Costley, C. D. (2002). When food becomes a drug: nonanabolic nutritional supplement use in athletes. The American Journal of Sports Medicine, 30(6), 907-916.

Da Silveira, C. L., de Souza, T. S. P., de Araújo Júnior, A. T., Batista, G. R., da Silveira Torres, M., do Socorro Cirilo-Sousa, M., \& Garrido, N. D. (2016). Níveis Bioquímicos após suplementação em Policias Militares durante treinamento físico. Motricidade, 12(1), 17-25.

Slater, G., Tan, B., \& Teh, K. C. (2003). Dietary supplementation practices of Singaporean athletes. International journal of sport nutrition and exercise metabolism, 13(3), 320-332.

Smith-Rockwell, M., Nickols-Richardson, S. M., \& Thye, F. W. (2001). Nutrition knowledge, opinions, and practices of coaches and athletic trainers at a division 1 university. International journal of sport nutrition and exercise metabolism, 11(2), 174-185.

Sobal, J., \& Marquart, L. F. (1994). Vitamin/mineral supplement use among athletes: a review of the literature. International Journal of Sport Nutrition and Exercise Metabolism, 4(4), 320-334.

Souza Júnior, T. P. D., Dubas, J. P., Pereira, B., \& Oliveira, P. R. D. (2007). Suplementação de creatina e treinamento de força: alterações na resultante de força máxima dinâmica e variáveis antropométricas em universitários submetidos a oito semanas de treinamento de força (hipertrofia). Revista Brasileira de Medicina do Esporte, 13, 303-309.

Sundgot-Borgen, J., Berglund, B., \& Torstveit, M. K. (2003). Nutritional supplements in Norwegian elite athletes-impact of international ranking and advisors. Scandinavian journal of medicine \& science in sports, 13(2), 138-144.

Tian, H. H., Ong, W. S., \& Tan, C. L. (2009). Nutritional supplement use among university athletes in Singapore. Singapore Medical Journal, 50 (2), 165.

Van Thuyne, W., Van Eenoo, P., \& Delbeke, F. T. (2006). Nutritional supplements: prevalence of use and contamination with doping agents. Nutrition research reviews, $19(1), 147-158$.

Volpe, S. L. (2007). Micronutrient requirements for athletes. Clinics in sports medicine, 26(1), 119-130.

Zeiser, C. C., \& Silva, R. D. (2007). O uso de suplementos alimentares entre os profissionais de educação física atuantes em academias da cidade de Florianópolis. Rev Nutr Pauta, 15(86), 30-3. 